\title{
Editorial
}

\section{The next generation of medical physicists}

In May 2013, a group of four students studying Medical Physics at the University of Warsaw, together with the help of several lecturers from various Polish Universities, organized a conference entitled "The Warsaw Medical Physics Meeting". Nearly 60 young physicists participated in the event and several interesting presentations were given by the young physicists and their professors.

After the conference, a proposal was made to publish the best of these lectures in Reports of Practical Oncology and Radiotherapy. The result is this supplement of RPOR, which you are reading right now. For most of these promising young physicists, this is the first paper they have ever published. As anyone who has ever spent time preparing and writing a manuscript knows, the process of writing a paper is more complex than it might at first appear. For this reason, these students were given expert guidance by their tutors. We believe that readers will agree with us when we commend the authors for the excellent results of their hard work.

The work of medical physicists typically involves the application of ionizing radiation in medicine and in that respect, all papers published in this supplement address aspects related to the medical application of ionizing radiation. What follows is a brief introduction to the papers included in this supplement.

Proton therapy is becoming increasingly important as new proton therapy centers open around the world each year. The first proton therapy center for deep tumors will open in Krakow next year (2015). In this sense, the paper written by Anna Dawidowska ${ }^{1}$ is timely. In her paper, Ms. Dawidowska describes the problem of energy deposition due to the $(p, n)$ reaction during proton therapy. Proton therapy is an emerging field within radiotherapy and it is important that young researchers develop expertise in this area. This paper is an excellent contribution to the field.

In our present era of Intensity Modulated Radiotherapy (IMRT), only very sophisticated methods of dose optimization are of interest, and this is as it should be. However, Marta Krystyna Giżyńska and Paweł Kukołowicz ${ }^{2}$ have written a well-developed article that provides a good overview of issues related to dose distribution optimization and we believe that this paper could be valuable educational material for lecturers in the field of radiotherapy.

Readers will also find two papers related to nuclear medicine. In the first paper, ${ }^{3}$ Krzysztof Kilian describes the current status and future perspectives of 68a-DOTA and analogs. This topic is relevant to all involved in nuclear medicine. The same author has also written a second article regarding the quality control process in the synthesis of 18F-Fludeoxyglucose. ${ }^{4}$ In that article, Kilian et al. share their experience in the quality control process to ensure that metallic impurities are reduced to an acceptable level. Quality control is one of the most important everyday tasks performed by medical physicists, and for this reason this article will interest all medical physicists, not just those who work in the field of nuclear medicine.

An important task of medical physicists is to accurately and reliably measure the radiation energy deposited in tissues. In radiotherapy, it is essential to be sure that the intended dose is delivered as expected. This process is checked by using beam monitor detectors. In an interesting paper included here, Konrad Nesteruk ${ }^{5}$ describes three new beam monitor detectors for ionizing radiation. These detectors can be used with various types of beams and for both hadrontherapy and radioisotope production.

Physicists are often involved in radiobiological research, especially given its relevance to medical radiotherapy. One important and highly relevant radiobiological phenomenon is the bystander effect. This effect is still incompletely understood, although several articles published in this journal have addressed this topic. ${ }^{6,7}$ In fact, in an upcoming issue of RPOR, a review article will address this topic in depth. Conflicting reports have been published regarding whether or not irradiated cells influence surrounding cells. In 1992, this phenomenon was described by Nagasawa and Little, ${ }^{8}$ although the underlying mechanisms for the bystander effect are still not fully elucidated. Interestingly, Urszula Kaźmierczak et al. ${ }^{9}$ investigated this effect in Chinese hamster ovary cells (CHO-K1) irradiated with high LET 12C ions 
and X-rays at the dose range of 0.1-4 Gy. As the authors report, no bystander effect was observed. Clearly, more studies will be needed before any definitive conclusions can be made regarding the bystander effect.

Finally, Marcin Pietrzak et al. have written a very interesting article entitled "Status report: nanodosimetry of carbon ion beams at HIL". ${ }^{10}$ Nanodosimetry is an emerging area of radiophysics which seeks to measure DNA damage to nanometric target volumes. Readers who are unfamiliar with this topic will find this article to be an excellent overview of the field, particularly as practiced at the Heavy Ion Laboratory (HIL) in Warsaw, Poland.

In conclusion, I believe that readers will be pleasantly surprised by the quality and relevance of the articles in this supplement. Enjoy!

\section{REFERENCES}

1. Dawidowska A, Ferszt MP, Konefał A. The determination of a dose deposited in reference medium due to $(p, n)$ reaction occurring during proton therapy. Rep Pract Oncol Radiother 2014;19:S3-8.

2. Giżyńska MK, Kukołowicz PF. Dose gradient based algorithm for beam weights selection in 3D-CRT plans. Rep Pract Oncol Radiother 2014;19:S9-12.

3. Kilian K. 68Ga-DOTA and analogues: current status and future perspectives. Rep Pract Oncol Radiother 2014;19:S13-21.

4. Kilian K, Chabecki B, Kiec J, et al. Synthesis, quality control and determination of metallic impurities
in18F-fludeoxyglucoseproduction process. Rep Pract Oncol Radiother 2014;19:S22-31.

5. Nesteruk KP. Beam monitor detectors for medical applications. Rep Pract Oncol Radiother 2014;19:S32-6.

6. Malicki J. The importance of accurate treatment planning, delivery, and dose verification. Rep Pract Oncol Radiother 2012;17:63-5.

7. Martin MJ, Zapatero J, Lopez M. Prevention of future incidents and investigational lines. Rep Pract Oncol Radiother 2011;16:153-61.

8. Nagasawa H, Little JB. Induction of sister chromatid exchanges by extremely low doses of alpha-particles. Cancer Res 1992;52:6394.

9. Kaźmierczak U, Banaś D, Braziewicz J, et al. Investigation of the bystander effect in CHO-K1 cells. Rep Pract Oncol Radiother 2014;19:S37-41.

10. Bantsar A, Pietrzak M, Jaskóła M, Korman A, Pszona S, Szefliński Z. Status report: nanodosimetry of carbon ion beam at HIL. Rep Pract Oncol Radiother 2014;19:S42-6.

Guest Editor

Pawel Kukolowicz Medical Physics Department, Centre of Oncology-Institute in Warsaw, Poland

E-mail address: pawel.kukolowicz@gmail.com http://dx.doi.org/10.1016/j.rpor.2014.04.010 1507-1367/@ 2014 Greater Poland Cancer Centre. Published by Elsevier Urban \& Partner Sp. z o.o. All rights reserved. 